\title{
Abschied von Gestern
}

$\mathrm{n}$ den letzten Jahren haben sich einige neuere allergologische Erkenntnisse verfestigt, die in diesem Heft zusammenfassend publiziert werden. Das Motto „Abschied von Gestern“ nach einem Film von Alexander Kluge aus dem Jahr 1966 - ist dabei keinesfalls überzogen. Bei genauerer Analyse ist der Abschied von Gestern vielmehr die Grundvoraussetzung für den Fortschritt, ja, für das Überleben der Allergologie, auch wenn damit schmerzhafte Einschnitte in unsere Denkgewohnheiten und eine gründliche Überarbeitung älterer Vorstellungen verbunden sind.

So lassen sich für die Allergieprävention, anders als noch vor Jahren angenommen, heute nur wenig gesicherte Erkenntnisse anbieten. Vor allem die strikte Allergenkarenz hat sich nicht als generell geeignete präventive $\mathrm{Maßnahme} \mathrm{erwie-}$ sen. Möglicherweise ist sogar der wohldosierte Allergenkontakt die Grundbedingung für eine Toleranz. Und möglicherweise sind von der Allergenexposition unabhängige Faktoren allergieprotektiv. Das Interview mit Prof. Erika von Mutius auf Seite 398 stellt diesen Sachverhalt eingehend dar.

Auch die spezifische Immuntherapie, seit Jahrzehnten und auch heute noch in den Leitlinien als einzige kausale antiallergische Maßnahmen apostrophiert, ist kei"Der Abschied von Gestern ist
die Grundvoraussetzung für
den Fortschritt, ja, für das
Überleben der Allergologie." neswegs eine generell überzeugende therapeutische Maßnahme. Die Wirkung bei allergischer Rhinokonjunktivitis ist begrenzt, bei allergischem Asthma fehlen letztlich überzeugende Daten für eine Effektivität, bei Neurodermitis stehen umfassende Studien noch aus. Auch hierzu finden sich in diversen Beiträgen in diesem Heftes nähere Informationen.

Die kritische Betrachtung der Allergologie aufgrund neuerer Erkenntnisse zwingt jeden von uns zu einer andauernden Auseinandersetzung mit den aktuellen Forschungsdaten. Hierzu stehen im September zwei wichtige Tagungen bevor. Da ist zunächst in Berlin vom 3. bis zum 6. September 2009 der 4 . Gemeinsame Allergiekongress der drei allergologischen Fachgesellschaften unter Leitung von Prof. Dr. Ulrich Wahn. Den Abstractband zu diesem Kongress erhalten Sie eingeschweißt zusammen mit dieser regulären Ausgabe des Allergo Journal als Sonderheft.

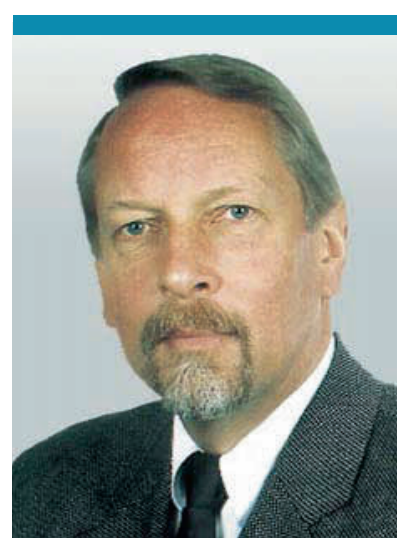

Prof. Dr. Gerhard SchultzeWerninghaus, Abteilung für Pneumologie, Allergologie und Schlafmedizin, Medizinische Klink III, BG-liches Universitätsklinikum Bergmannsheil, Bochum

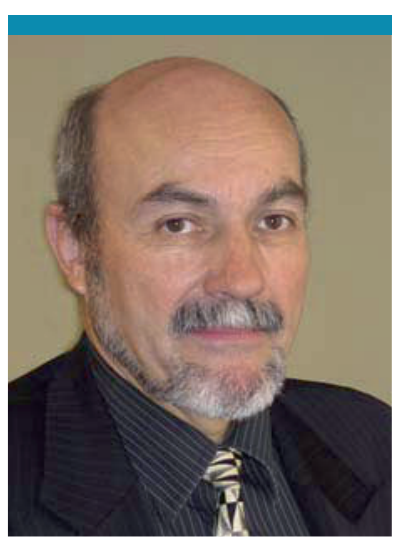

Prof. Dr. Dr. Johannes Ring, Klinik und Poliklinik für Dermatologie und Allergologie am Biederstein, Technische Universität München

Es folgt vom 23. bis zum 26. September in Davos der 25. Fortbildungskongress „Fortschritte der Allergologie, Dermatologie, Pneumologie und Immunologie" unter Leitung von Prof. Dr. Dr. Johannes Ring. Die Abstracts dazu finden Sie in diesem Heft ab Seite 420. Im Fokus stehen in Davos dieses Jahr Autoimmunerkrankungen, welche interessanterweise im 20. Jahrhundert ähnliche Zuwachsraten an Prävalenz zeigten wie die Allergien. Die Rolle von Immunreaktionen bei Krebserkrankungen ist vom Konzept der „Immun-Überwachung" weit fortgeschritten zu immunologischen Therapieformen und Vakzinierungsversuchen. Weitere Schwerpunkte sind berufsbedingte Allergien und das Thema „Klimawandel und Allergie“. Darüber hinaus deckt dass Programm klassische allergologische Probleme ab wie Arzneimittelallergie, Photoallergie oder Unterschiede zwischen Haut- und Atemwegen in der Allergieentstehung und -ausprägung.

Die Herausgeber hoffen auf das rege Interesse der Leser des Allergo Journal an dem Fortbildungsangebot. Kommentare und Kritik sind in Form von Leserbriefen jederzeit willkommen.

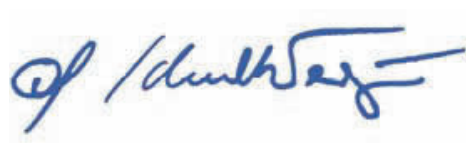

Prof. Dr. G. Schultze-Werninghaus

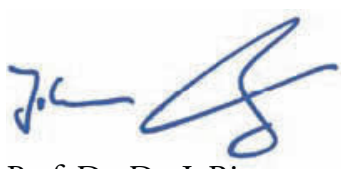

Prof. Dr. Dr. J. Ring 